\title{
Overview of the Gas and Aerosol Metrology
}

\author{
C. J. Tsai ${ }^{1 *}$ and S. G. Aggarwal ${ }^{2 *}$ \\ ${ }^{1}$ Institute of Environmental Engineering, National Chiao Tung University, Hsinchu 30010, Taiwan \\ ${ }^{2}$ Analytical Chemistry, CSIR-National Physical Laboratory, New Delhi 110012, India \\ Published online: 11 October 2013 \\ (C) Metrology Society of India 2013
}

\begin{abstract}
There are several exciting developments noted in gas and aerosol measurements in recent years. On the other hand not much work has been done on the calibration facility, traceable standards, and certified reference materials for this field of science. More attention and concerns are needed for the comparable and SI traceable data quality in gas and aerosol measurements. The aim of this special issue of MAPAN-Journal of Metrology Society of India is to highlight such issues.
\end{abstract}

Keywords: Gas and aerosol metrology; Calibration standards; Traceability; Nanoparticle measurement; Air quality and vehicular emission regulations

\section{Introduction}

In aerosol instrumentation field, a significant development can be seen in the last 10-15 years. Now specific instruments are available for the measurement of nanoparticle characteristics, particle number distribution, new particle formation and growth, cloud condensation nuclei activity, black carbon and radiative/scattering properties, single particle and in situ aerosol chemistry, etc. Although the new instrumental techniques are very helpful to better understand the aerosol physics and chemistry, it is not easy to obtain the calibration standards, reference materials and methods at the laboratory level to check the data quality regularly. These instruments are often calibrated at manufacturing site only. Therefore, working on instrumental calibration, standards and methods is equally important and needs more attention. In addition, particles have adverse health effects and thus constitute an important component of the National Ambient Air Quality Standards (NAAQS) in many countries around the world. For precise and accurate air quality data, it is very important that the instruments deployed for these measurements should be calibrated with the SI traceable standards. Funding agencies should be aware of this issue, and accept the results from the principle investigators and research teams only

*Corresponding author, E-mail: cjtsai@mail.nctu.edu.tw; aggarwalsg@ nplindia.org when their results have the traceability link to SI. The regulatory authorities/ministry should work together with national metrology institutes (NMIs) to improve the data quality of ambient measurements. This will offer great help to better make the policies and decisions on the mitigation of related impacts.

Aiming these objectives and to enhance the awareness of current developments on gas and aerosol measurement techniques, calibration, standards with the importance of SI traceable data quality, a "one-day pre-AdMet workshop on gas and aerosol metrology" was organized at National Physical Laboratory, New Delhi, India on February 20th, 2013. About 80 participants (students, technical persons, scientists, teachers and people from industries) attended the workshop including 6 invited delegates from Japan, Taiwan, UK and USA.

This special issue of MAPAN-Journal of Metrology Society of India is aimed to highlight the need of standards and traceable calibration in ambient measurements, uncertainty in related measurements, new design/method and theoretical comparison in aerosol measurements, etc. This issue comprises the papers from the distinguished groups on these topics (which include the talk given in oneday pre-AdMet workshop) under the title, "Aerosol Measurement Science and Technology".

In this special issue, the first paper is from Dr. Paul Quincey, NPL, UK [1]. This discusses about regulatory measurements of particle size, number and mass concentration in air, and related metrological issues. Authors mentioned that generally the metrological aspects have 
been covered by the monitoring network operators, with a little involvement from NMIs. The paper outlines some of the key metrological problems in this area, and the current position in dealing with them from a UK and European perspective. More involvement of NMIs in air quality measurement is recommended.

In the next paper, Dr. Shankar G. Aggarwal, NPL, India [2] highlighted the traceability issue of size cutoff of $\mathrm{PM}_{2.5}$ and $\mathrm{PM}_{10}$ samplers. Authors emphasised that the traceability in particle size and mass is either missing or poorly followed by the manufacturers and users. This is a significant factor for less reliable data in air quality measurements related to PM. NMIs has to take initiatives for setting up the PM calibration facilities. NMIs should also work on industrial participation in particle metrology program.

The paper from Dr. John G. Watson and Judith C. Chow of DRI in USA [3] discusses about the need of measurement standards for flow rate/volume and gas concentrations, which are essential components of any air quality monitoring program. Transfer standards are most often used for calibration, performance testing, and auditing of field monitors. These must be traceable to primary standards. Authors mentioned that several methods are available for generating these standards and tracing them to fundamental primary standards. These methods are not necessarily equivalent, and compensation must be made for differences between the gas mixtures and the operating environments.

Dr. Yury A. Kustikov, D.I. Mendeleyev Institute for Metrology, Russia [4] reported the progress in development of Russian national measurement standards in the field of mass concentration measurement of suspended particles. This paper describes the state primary special standard GET 164 and the specificity of ensuring the traceability in disseminating this primary special standard to the working measurement instruments. Methods used to generate aerodispersed media with desired properties and measurements are validated. Challenges and recommendations for achieving the highest accuracy in the measurements related to the primary special standard GET 164 are discussed.

Dr. M.V.S.N. Prasad, NPL, India [5] reviewed the existing facility for atmospheric parameter measurements at Radio \& Atmospheric Sciences Division (RASD) of CSIR-National Physical Laboratory, India. The paper also discusses the recent data observed based on the current facility. They have also mentioned about the forthcoming observatory, which has to be built in western Himalayan region. This station and instrumentation setup will be used in future for long-term atmospheric measurements as well as to organize short-term campaign by different groups.

Aerosol instruments are usually designed for measuring spherical particles. However, many nanoparticles are in the form of agglomerates or aggregates. Prof. C.J. Tsai, NCTU, Taiwan [6] discussed in his paper about the effect of particle morphology on the mobility diameter measurement of the EEPS in which a unipolar diffusion charger was implemented. The SMPS was used in parallel as a reference method. The open agglomerates acquired more charges than that of spheres with the same mobility diameter due to the higher electrical capacitance. This led to the sizing difference between SMPS and EEPS for partly sintered silver aggregates and agglomerates. Theoretical calculation was carried to support the observation in the experiments.

The paper by Dr. Da-Ren Chen, Virginia Commonwealth University, USA [7] describes a design and evaluation of a compact nanoparticle sampler. The sampler design is very compact, and the provided data allows applying it to the relevant research tasks. They have investigated the collections efficiency $(>90 \%)$ for particles ranging from 50 to $500 \mathrm{~nm}$. A careful parametric study was performed for the optimum operational condition of the sampler. The charging performance for particles of different materials was also studied.

In another paper, Dr. A. Lloyd, International Council on Clean Transportation, USA [8] describes the importance of emission measurements from transportation, quality of fuel and related policy making in India. Using examples where real world emissions of $\mathrm{NO}_{\mathrm{x}}$ exceed those obtained during vehicle emission test procedures, recommendations are made for India in designing and applying an air quality management (AQM) system, which relates emissions, meteorology and atmospheric chemistry to ambient air quality concentrations. Specific recommendations are made to require Euro 6/VI vehicle emission standards, nationwide ultra low sulfur fuels, controls of evaporative emission during refueling, improved initial vehicle emission testing, routine testing of fuels, and an enforceable vehicle in use emission testing program.

In summary, this special issue comprises articles which are focused on precise and accurate measurements of gas and aerosol particles, the important parameters of air quality standards. We thank all authors, co-authors and reviewers in this issue, Managing Editor and publication team of the journal for their contributions and help to make this issue possible. We are grateful to the Editor-in-Chief and the Publication and Information Committee of MSI for inviting us as the guest editors for this special issue. We hope that the coverage of the material on the topic would be of great interest to the scientific community, and reader will enjoy reading related papers at one place in this special issue.

\section{References}

[1] P. Quincey, S. Beccaceci, D. Butterfield, D. Sarantaridis and J. Tompkins, Aerosol metrology supporting air quality monitoring in the United Kingdom and Europe, MAPAN-J. 
Metrol. Soc. India, 28(3) (2013) 145-152. doi: 10.1007/s12647013-0061-1.

[2] S.G. Aggarwal, P. Mandal, B. Sarangi, K. Singh, J. Pokhariyal, S.K. Mishra, S. Agarwal, D. Sinha, S. Singh, C. Sharma and P.K. Gupta, Traceability issue in $\mathrm{PM}_{2.5}$ and $\mathrm{PM}_{10}$ measurements, MAPAN-J. Metrol. Soc. India, 28(3) (2013) 153-166. doi: 10.1007/s12647-013-0073-x.

[3] J.G. Watson, J.C. Chow, R.J. Tropp, X. Wang, S.D. Kohl and L.W.A. Chen, Standards and traceability for air quality measurements: flow rates and gaseous pollutants, MAPAN-J. Metrol. Soc. India, 28(3) (2013) 167-179. doi: 10.1007/s12647-013-0071-z.

[4] Y.A. Kustikov and B.I. Popov, Progress in development of Russian national measurement standards in the field of mass concentration measurement of suspended particles, MAPAN-J. Metrol. Soc. India, 28(3) (2013) 181-191. doi:10.1007/s12647013-0077-6.

[5] M.V.S.N. Prasad, C. Sharma, B.C. Arya, T.K. Mandal, S.N. Singh, M.J. Kulshrestha, R. Agnihotri, S.K. Mishra and S.K. Sharma, Experimental facilities to monitor various types of atmospheric parameters in the Radio \& Atmospheric Sciences Division (RASD) of CSIR-National Physical Laboratory, MAPAN-J. Metrol. Soc. India, 28(3) (2013) 193-203. doi: 10.1007/s12647-013-0067-8.

[6] A. Awasthi, B.S. Wu, C.N. Liu, C.W. Chen, S.N. Uang and C.J. Tsai, The effect of nanoparticle morphology on the measurement accuracy of mobility particle sizers, MAPAN-J. Metrol. Soc. India, 28(3) (2013) 205-215. doi: 10.1007/s12647-013-0068-7.

[7] H. Jing, S. He, Q. Ou, T.C. Hsiao and D.R. Chen, Development of a compact electrostatic nanoparticle sampler for offline aerosol characterization, MAPAN-J. Metrol. Soc. India, 28(3) (2013) 217-226. doi: 10.1007/s12647-013-0072-y.

[8] A.C. Lloyd and G. Bansal, Emissions from transportation and fuels: the importance of initial and real world measurements for policy making in India, MAPAN-J. Metrol. Soc. India, 28(3) (2013) 227-234. doi: 10.1007/s12647-013-0065-x.

\section{Author Biographies}

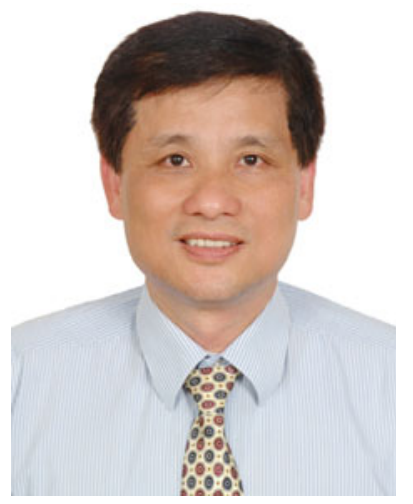

C. J. Tsai is the coordinator of Environmental Engineering Program of Taiwan National Science Council, and distinguished professor of the Institute of Environmental Engineering, Chiao Tung University, Hsinchu, Taiwan. He graduated from Mechanical Engineering Department, National Taiwan University, and received his MS and Ph.D. in the Particle Technology Laboratory (PTL), Mechanical Engineering Department, University of Minnesota. From 1990,
Nanoparticle and Air Quality Laboratory, in Taiwan to conduct researches on nanoparticle and aerosol measurement and instrumentation, aerosol physics, air pollution control devices and air quality. He has published a total of 150 peer-reviewed papers and has been granted for 40 patents. In 2004-2007 and 2012-2015, he received distinguished research awards from Taiwan NSC (National Science Council), and in 2006, he was bestowed the prestigious International Aerosol Fellow Award from the IARA (International Aerosol Research Assembly). Dr. Chuen-Jinn is one of the key founding members of TAAR (Taiwan Association for Aerosol Research), and AARA (Asian Aerosol Research Assembly). He is now one of the two editors-in-chief of AAQR (Aerosol and Air Quality Research, http://aaqr.org/), the SCI journal published by TAAR. He has served in the aerosol community as the vice-president and president of AARA, the editorial board member of JAS and the editor of AST.

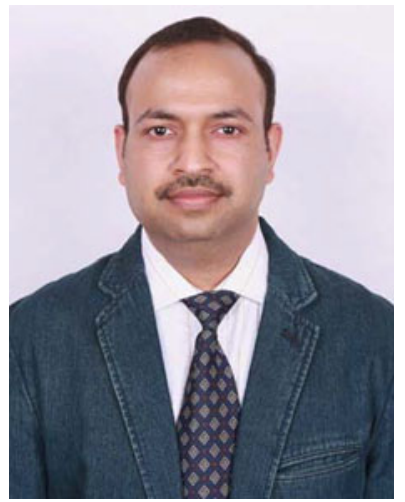

S. G. Aggarwal did his Ph.D. in Analytical Chemistry with Prof. K.S. Patel from Pt. Ravishankar Shukla University, Raipur (CG), India in 1999. After his Ph.D. award, he worked with Prof. C. J. Tsai at NCTU, Taiwan and ITRI, Taiwan for several years on gas and aerosol related researches (measurement and control technology). Thereafter he received JSPS fellowship and worked at Hokkaido University, Japan with Prof. K. Kawamura on aerosol chemistry and its microphysical properties before he joined CSIR-National Physical Laboratory, New Delhi, India in 2009. He is working as senior scientist, and his current research focus at NPL is "metrology for national ambient air quality standards (NAAQS)" in which he involves in preparation of aqueous standards, preparation of gas standards and PM calibration. So far he has published more than 45 papers in high ranked peer-reviewed journals in the field of analytical chemistry and aerosol chemistry/physics and measurements. He has received several awards including best research team member in ITRI, outstanding poster in AAC2011, is reviewer of several international journals, editorial board member of AAQR.

Dr. Chuen-Jinn has established one of the best aerosol laboratories, 